\title{
Caractérisations Hydroclimatiques et Estimation du Taux de Recharge de la Nappe Libre du Haut Bassin Versant de la Korama, Commune de Droum/Région de Zinder/Niger
}

\author{
Issa Malam Salmanou Souleymane, \\ Université de Zinder, Faculté des Sciences et Techniques, \\ Département des Sciences Géologiques et Environnementales, Zinder, Niger \\ Maman Sani Abdou Babaye, \\ Université Dan Dicko Dankoulodo, Faculté des Sciences et Techniques, \\ UMR SERMUG, Département de Géologie, Maradi, Niger
}

\section{Illias Alhassane,}

Université d'Agadez, Faculté des Sciences et Techniques,

Département de Géologie, Agadez, Niger

\section{Sandao Issoufou, \\ Ousmane Boureima,}

Université Abdou Moumouni de Niamey, Faculté des Sciences et

Techniques, Département de Géologie, Niamey, Niger

\section{Résumé}

La zone d'étude, la Commune de Droum, est située dans le haut bassin versant de la Korama. Celle-ci dispose de plusieurs aquifères, dont l'aquifère phréatique des sables récents ayant des hautes potentialités en eau souterraine. Cet aquifère est très sollicité pour l'approvisionnement en eau de boisson, l'abreuvage des animaux et pour l'irrigation en pleine expansion. Cependant, cet aquifêre peu profond reste très sensible à la variabilité climatique ayant affecté la région, comme le reste du sahel, de 1970 à nos jours. L'objectif principal de cette étude est de caractériser les paramètres climatiques puis de quantifier la recharge de cette nappe pendant la période de 1921-2016. La méthodologie adoptée est la caractérisation des différents paramètres climatiques et l'estimation du bilan hydrique à partir de plusieurs techniques (l'indice pluviométrique annuel, le filtre passe-bas de Hanning et les tests statistiques de détection de rupture tels que le Test de Petitt, procédure bayésienne de Lee et Heghinian, segmentation d'Hubert, méthodes Thornthwaite et Penman pour le calcul du bilan) ayant permis d'aboutir à plusieurs résultats. Ainsi, l'analyse de la série pluviométrique montre 
l'alternance de trois phases: la phase humide de 1921 à 1964, la phase sèche de 1965 à 1997 et la phase normale de 1998 à 2016, et une rupture significative traduisant la baisse de précipitations à partir de 1965 et peu significative mettant en évidence le retour de celles-ci à partir de 1998. Par ailleurs, le bilan hydrologique met en évidence le comportement de la nappe pour ces différentes phases climatiques. On observe, cependant que la recharge de cette nappe en période sèche est relativement faible par rapport à la période humide. Celle-ci provienne probablement de la recharge indirecte car il y a eu une intensification de ruissellement. A partir de 1998, la tendance observée du retour des précipitations a eu comme conséquence, une légère augmentation de la lame d'eau infiltrée par rapport à la période sèche. Toutefois, celle-ci est trop faible quand on le compare à celle de la période humide et aux demandes accrues de cette ressource pour les différents usages.

Mots clés : Commune De Droum, Paramètres Climatiques, Région De Zinder, Bilan Hydrologique, Tests Statistiques 


\title{
Hydroclimatic Characterisation and Assessment of Recharge Rate in the Upper Korama Watershed, Droum Rural District, Zinder Region, Niger
}

\author{
Issa Malam Salmanou Souleymane, \\ Université de Zinder, Faculté des Sciences et Techniques, \\ Département des Sciences Géologiques et Environnementales, Zinder, Niger \\ Maman Sani Abdou Babaye, \\ Université Dan Dicko Dankoulodo, Faculté des Sciences et Techniques, \\ UMR SERMUG, Département de Géologie, Maradi, Niger \\ Illias Alhassane, \\ Université d'Agadez, Faculté des Sciences et Techniques, \\ Département de Géologie, Agadez, Niger \\ Sandao Issoufou, \\ Ousmane Boureima, \\ Université Abdou Moumouni de Niamey, Faculté des Sciences et \\ Techniques, Département de Géologie, Niamey, Niger
}

\begin{abstract}
The study area, the municipality of Droum, is located in the high watershed of Korama. It has several aquifers, including the aquifer of recent sands with high potential in groundwater. This aquifer is in great demand for the supply of drinking water, animal watering and for expanding irrigation. However, this shallow aquifer remains very sensitive to the climate variability that has affected the region, like the rest of the Sahel, from 1970 to the present day. The main objective of this study is to characterize the climate parameters and quantify the recharge of this aquifer during the period of 1921-2016. The methodology adopted is the characterization of the various climatic parameters and the estimation of the water balance from several technics (annual pluviometric index, Hanning's low-pass filter and statistical tests for detecting discontinuities such as it is: Petitt's test, Lee and Heghinian's bayesian procedure, Hubert's segmentation procedure, Thornthwaite and Penman's methods for water balance calculate) that have led to several results. Thus, the analysis of the rainfall series shows the alternation of the three phases : the wet phase from 1921 to 1964 , the dry phase from 1965 to 1997 and the normal phase from 1998 to 2016, and a significant discontinuities reflecting the decrease in rainfall starting at 1965 and not very significant
\end{abstract}


highlighting the return of these from 1998. Moreover, the hydrological balance highlights the behavior of the water table for these different climatic phases. It is observed, however, that the recharge of this layer in the dry period is relatively low compared to the wet period. This probably comes from indirect recharge because there has been an intensification of runoff. From 1998, the observed trend of precipitation return has resulted in a slight increase in the infiltrated water slide compared to the dry period. However, it is too weak compared to that of the wet period and the increased demands of this resource for different uses.

Keywords : Droum Commune, Climate Parameters, Zinder Region, Water Balance, Statistical Tests

\section{Introduction}

La Commune de Droum, zone aride à semi-aride, située dans la region de Zinder, centre de la partie Est du Niger, est confrontée depuis plusieurs années au phénomène de changement climatique. Ce changement se caractérise principalement par des sècheresses récurrentes et la modification du régime pluviométrique depuis la décennie 1970-1980. Cette modification peut avoir des incidences sur le régime hydrologique et sur les ressources en eau souterraine de son bassin versant. En effet, nombreux d'auteurs, à travers le monde, ont mis en évidence l'impact de la variabilité climatique sur les ressources en eaux (Bi Tié et al., 2006; Saley et al., 2010) et particulièrement sur la recharge des eaux souterraines (Scanlon et al., 2006). Dans les régions du Sud-Ouest Américain, la variabilité climatique se traduise par une recharge jusqu'à trois fois supérieure pendant les périodes de l'oscillation australe EL Niño (ENSO) que pendant les périodes dominées par la Niñas. L'étude de la variabilité climatique aux échelles décennales, en Afrique, donne lieu à de taux de recharge de $30 \mathrm{mn} / \mathrm{an}$ pendant la sècheresse du Sahel (1970-1986) à 150 $\mathrm{mm} / \mathrm{an}$ pendant les périodes de non sècheresse (Scanlon et al., 2006). C'est dans cette perspective que s'inscrit cette étude, dont l'objectif principal consiste en la caractérisation hydroclimatique et l'élaboration du bilan hydrologique du haut bassin versant de la Korama dans la Commune de Droum, région de Zinder. Spécifiquement, cette étude vise à :

- caractériser les principaux paramètres climatiques de la zone d'étude;

- estimer la part de la pluie efficace annuelle sur la période de 1961 à 2013 à travers différentes méthodes de calcul du bilan hydrologique;

- voir les impacts de ces changements sur les ressources en eau souterraine et de surface. 


\section{Présentation de la zone d'étude}

La Commune de Droum, zone d'étude occupe le haut bassin versant de la Korama, située au Sud de la région de Zinder dans le département de Mirriah, s'inscrit entre $13^{\circ} 23^{\prime}$ et $13^{\circ} 48^{\prime}$ de latitude Nord et $8^{\circ} 39^{\prime}$ et $9^{\circ} 1^{\prime}$ de la longitude Est (fig.1). Elle couvre une superficie de $870 \mathrm{~km}^{2}$, avec une population estimée à 102.306 hbts (INS, 2010), dont la densité est de $118 \mathrm{hbts} / \mathrm{km}^{2}$ (ONG Karkara, 2015). L'agriculture constitue la principale activité économique des populations, avec un degré d'utilisation de sol de près de 93,3\%, soit l'un des plus élevés au Niger (SNV, 2014). L'élevage occupe la seconde place. Le climat du bassin est de type sahélien, caractérisé par deux saisons: une saison des pluies de Juin à Septembre et une saison sèche d'Octobre à Mai. Le module pluviométrique inter annuel sur la période de 1921-2014 est de $344 \mathrm{~mm}$. Sur le plan géologique, la Commune est marquée au Nord-Est par des formations du Précambrien de Damagaram, comprenant des granites hyperalcalins, des schistes, des micaschistes et des gneiss; à l'Ouest les formations sédimentaires constituées de grès argileux du $\mathrm{CI} / \mathrm{H}$ et au Sud les alluvions récentes de la Korama, extrémité occidentale du bassin du Lac Tchad. Sur le plan hydrogéologique, il existe trois aquifères dans le haut bassin de la Korama, qui sont: l'aquifère de socle du Damagaram, l'aquifère multicouche $\mathrm{du} \mathrm{CI} / \mathrm{H}$ et l'aquifère des alluvions du Quaternaire et des sables récents de la Korama. Ce dernier aquifère renferme l'essentiel de la ressource en eau pour l'approvisionnement de la population. Cependant, face à la variabilité climatique que connait la région depuis plusieurs années, il s'avère nécessaire d'entreprendre une étude afin de pouvoir cerner l'impact de ce dernier sur la ressource en eau. 


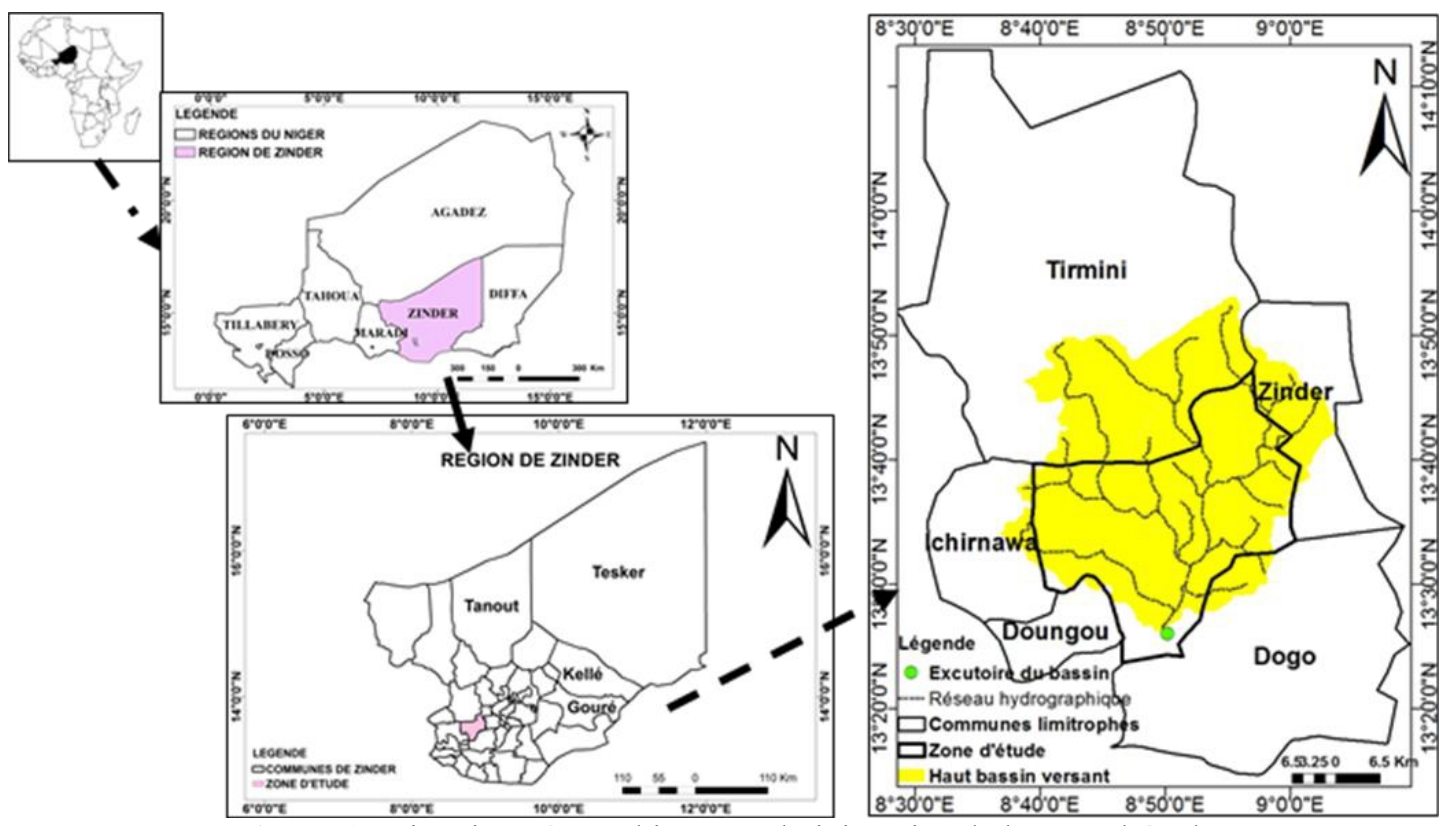

Figure 1 : Situation géographique et administrative de la zone d'étude

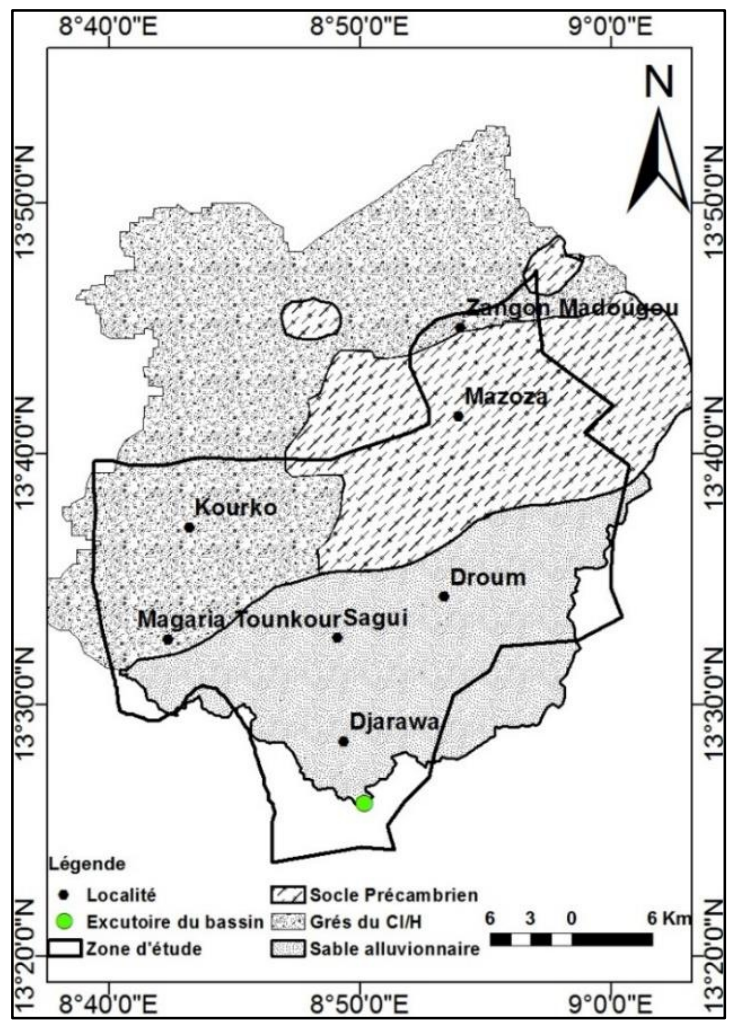

Figure 2 : Géologie de la zone d'étude, CI/H : Continental Intercalaire/Hamadien 


\section{Matériel et méthodes}

\section{II.2. Matériel}

Le matériel comprend les données et les outils suivants :

$\checkmark$ Les données sont :

- les paramètres climatiques (les hauteurs pluviométriques, la température maximum, la température minimum et l'évapotranspiration potentielle (ETP de Penman) de la station synoptique de Zinder Aéroport ;

- les rapports existants sur l'hydrologie et l'hydrogéologie dans la zone d'étude;

- les cartes topographiques et géologiques ;

- les chroniques hydrométriques couvrant la période de 1969 - 1981 et de 1994 - 2016. Elles concernent, pour la période de 1969-1981, les volumes d'eau écoulées et les valeurs de coefficients de ruissellement, tandis que pour la période de 1994 - 2016, il s'agit uniquement des volumes d'eau écoulées.

Les outils utilisés sont constitués principalement par des logiciels composés par : ArcGis et Khronostat.

\section{II.3. Méthodes}

Les paramètres hydroclimatiques sont issus de la station synoptique de Zinder. Le choix de cette station a été motivé par le fait qu'elle dispose d'un nombre important de variables climatiques, fiables et portant sur une longue période. En plus, on peut considérer que la station n'est pas éloignée de la zone d'étude. L'ensemble de ces données ont été obtenues au niveau du centre agro hydrométéorologique (AGRHYMET). Enfin, les chroniques hydrométriques sont issues de la station de jaugeage de Koutchika, obtenues auprès de la direction de l'hydraulique et de l'assainissement de Zinder (DRH/A).

\section{II.3.1. Traitements de données}

\section{II.3.1.1. Variabilité de la pluviométrie}

L'approche méthodologique adoptée pour les traitements des données hydroclimatiques porte sur les aspects suivants: l'indice pluviométrique annuel, le filtre passe-bas de Hanning et les tests statistiques de détection de rupture (Test de Petitt, procédure bayésienne de Lee et Heghinian, segmentation d'Hubert).

\section{$\checkmark$ Détermination de l'Indice pluviométrique}

L'indice pluviométrique permet d'apprécier l'évolution de la pluviométrie au cours des différentes années de la période d'étude. En effet, il permet d'observer la variabilité interannuelle des précipitations afin d'identifier les années déficitaires et excédentaires. Lorsque la pluviométrie 
moyenne annuelle est inférieure, égale ou supérieure à la pluviométrie moyenne de la série, les indices pluviométriques négatifs, nuls à faibles ou bien positifs mettent en évidence respectivement les années sèches, normales ou humides (Abdou Babaye, 2012).

Il se définit comme une variable centrée réduite exprimée par l'équation de Nicholson suivante:

Avec

$$
\mathrm{I}=\frac{x i-\bar{x}}{\sigma}
$$

- $\quad \boldsymbol{x} \boldsymbol{i}$ pluviométrie de l'année i ;

- $\quad \bar{x}$ pluviométrie moyenne interannuelle sur la période de référence ;

- $\boldsymbol{\sigma} \square \square$ écart-type de la pluviométrie interannuelle sur la période de référence.

\section{- Filtre Passe-Bas de Hanning}

La méthode filtre passe-bas de Hanning d'ordre 2 permet d'éliminer les variations saisonnières dans une série chronologique. Cette méthode vient compléter le Test de Petitt. Le calcul des totaux pluviométriques pondérés est effectué au moyen des équations suivantes présentées par Assani (1999):

$\mathrm{X}_{(\mathrm{t})}=0.06 \mathrm{X}_{(\mathrm{t}-2)}+0.25 \mathrm{X}_{(\mathrm{t}-1)}+0.38 \mathrm{X}_{(\mathrm{t}-2)}+0.25 \mathrm{X}_{(\mathrm{t}+1)}+0.06 \mathrm{X}_{(\mathrm{t}+2)} \quad$ pour $3 \leq \mathrm{t} \leq(\mathrm{n}-2)$

Où $\mathrm{X}_{(\mathrm{t})}$ est le total pluviométrique pondéré du terme $\mathrm{t}, \mathrm{X}_{(\mathrm{t}-2)}$ et $\mathrm{X}_{(\mathrm{t}-1)}$ sont les totaux pluviométriques observés de deux termes qui précèdent immédiatement le terme $t$ et $X_{(t+2)}$ et $X_{(t+1)}$ sont les totaux pluviométriques observés de deux termes qui suivent immédiatement le terme t. Pour plus de détail, nous recommandons le lecteur aux travaux d'Assani (1999).

\section{- Tests statistiques de détection de rupture}

Dans le cadre de cette étude, trois méthodes statistiques ont été retenues pour leur puissance et pertinence ainsi que leur utilisation courante dans de nombreux travaux (Soro et al., 2006). Il s'agit :

\section{$\rightarrow$ Test de Petitt}

Le test de Petitt (1979) est un test non-paramétrique qui dérive de la formulation du test de Mann-Whitney (Paturel et al., 1997 ; Fita et al., 2015). Il consiste à découper la série principale (x) de $\mathrm{N}$ éléments en deux sous séries $\left(x_{i}\right.$ et $\left.x_{j}\right)$ à chaque instant $t$ compris entre 1 et $\mathrm{N}-1$. L'absence d'une rupture dans cette série constitue l'hypothèse nulle $\mathrm{H}_{0}$. Sa mise en œuvre suppose que, pour tout instant $t$ compris entre 1 et $\mathrm{N}$, les séries chronologiques $\left(\mathrm{X}_{\mathrm{i}}\right) \mathrm{i}=1$ à $\mathrm{t}$ et $\mathrm{t}+1$ à $\mathrm{N}$ appartiennent à la même population. Si l'hypothèse nulle est rejetée, une estimation de la date de rupture est donnée pour l'instant $t$, définissant le maximum de la valeur absolue de la variable $\left(\mathrm{U}_{\mathrm{tN}}\right)$.

Petitt a définit la variable $\mathrm{U}_{\mathrm{t}, \mathrm{N}}$ (Paturel et al., 1997) : 


$$
\mathrm{U}_{\mathrm{t}, \mathrm{N}}=\sum_{i=1}^{t} \sum_{J=t+1}^{N} D i j
$$

où $\operatorname{Dij}=\operatorname{sgn}\left(x_{i}-X_{j}\right)$ avec $\operatorname{sgn}(Z)=1$ si $Z>0,0$ si $Z=0$ et -1 si $\mathrm{Z}<0$

Il propose de tester l'hypothèse nulle en utilisant la statistique $K_{N}$ définie par le maximum en valeur absolue de $\mathrm{U}_{\mathrm{t}, \mathrm{N}}$ pour $\mathrm{t}$ variant de 1 à $\mathrm{N}-1$.

A partir de la théorie des rangs, Petitt montre que si k désigne la valeur de $\mathrm{K}_{\mathrm{N}}$ prise sur la série étudiée, sous l'hypothèse nulle, la probabilité de dépassement de la valeur $\mathrm{k}$ est donnée approximativement par:

$$
\operatorname{Prob}\left(K_{N}>k\right) \gg 2 \exp \left(-6 k^{2} /\left(N^{3}+N^{2}\right)\right)
$$

Pour un risque $\alpha$ de première espèce donnée, si la probabilité de dépassement estimée est inférieure à $\alpha$, l'hypothèse nulle est rejetée. La série comporte alors une rupture localisée au moment $\tau$ où est observé $\mathrm{K}_{\mathrm{N}}$.

Les données utilisées dans le cadre de cette étude s'étendent sur une période de 93 ans. Ceci est en accord avec les exigences de l'OMS (1980) qui explique qu'il faut un minimum de 30 ans pour qu'une étude soit valable (Kanohin et al., 2009).

\section{$\rightarrow$ Procédure bayésienne de Lee et Heghinian}

Contrairement au Test de Petitt, cette méthode propose une approche paramétrique. Son application nécessite une distribution normale des valeurs de la série (Paturel et al., 1995 et 1997; Sene et Ozer., 2002). La méthode bayésienne de Lee et Heghinian (1977) vise à confirmer ou à infirmer l'hypothèse d'un changement de moyenne dans la série (Soro et al., 2006). L'absence de rupture dans la série constitue l'hypothèse nulle. La procédure repose sur le modèle suivant :

$$
X_{i}= \begin{cases}\mu+\varepsilon_{i} & i=1, \ldots, \tau \\ \mu+\delta+\varepsilon_{i} & i=\tau+1, \ldots, N\end{cases}
$$

Où les $\varepsilon_{\mathrm{i}}$ sont indépendants et normalement distribués, de moyenne nulle et de variance $\sigma$.

Les variables $\boldsymbol{\tau}, \boldsymbol{\mu}, \boldsymbol{\delta}$ et $\boldsymbol{\sigma}$ sont des paramètres inconnus. $\boldsymbol{\tau}$ et $\boldsymbol{\delta}$ représentent respectivement la position de la rupture dans le temps et l'amplitude du changement sur la moyenne. La méthode fournit donc la probabilité que la rupture se produise au moment $\boldsymbol{\tau}$ dans une série où on suppose à priori qu'il y a effectivement un changement à un moment indéterminé.

\section{$\rightarrow$ La procédure de segmentation d'Hubert (Hubert et al., 1989)}

Plusieurs auteurs ont utilisé cette méthode dans l'étude de la variabilité climatique : Hubert et al., (1989) ; Lubes-Niel et al., (1998) ; Sandra (2004); Fita et al., (2015). La procédure de segmentation a pour principe le découpage d'une série en $m$ segments de telle sorte que la moyenne calculée sur tout 
segment soit nettement différente de la moyenne du (des) segment (s) voisin (s).

La segmentation est retenue lorsque l'écart quadratique entre elle et la série est minimum. Cette condition est nécessaire mais non suffisante pour la détermination de la segmentation optimale (Kingumbi et al., 2000). Il faut lui adjoindre, par application du test de Scheffé, la contrainte selon laquelle les moyennes des deux segments contigus doivent être significativement différentes.

\section{- Etudes des autres paramètres climatiques}

Concernant les autres paramètres climatiques qui sont entre autre la température et l'évapotranspiration potentielle (ETP) le traitement portera sur :

- la détermination de moyenne arithmétique, l'écart type, le maximum, le minimum ;

- et l'élaboration des différents graphiques

\section{II.3.3.2. Estimation de la recharge}

\section{- Détermination de l'ETP par la méthode de Thornthwaite}

Plusieurs méthodes plus ou moins complexes sont utilisées pour calculer l'évapotranspiration potentielle: Thornthwaite, Turc, Coutagne, Penman, FAO 56 Penman-Monteih, Havregas. Elles tiennent compte des mesures climatologiques telles que les températures de l'air, les humidités, la radiation solaire globale, la vitesse du vent (Abdou Babaye, 2012 ; Hamadi et Chiraz, 2003 in Bouteldjaoui et al., 2011). Dans le cadre de ce travail l'estimation de ce paramètre a été faite à l'aide de la méthode de Thornthwaite. Cette méthode est basée sur la température de l'air :

$\operatorname{ETP}_{(\mathrm{mm})}=16\left(\frac{10 T}{I}\right)^{\mathrm{a}} \cdot \mathrm{F}(\lambda)$

Avec:

$$
\mathrm{I}=\sum_{n=1}^{12} \mathrm{i}(14) \mathrm{i}=\left(\frac{T}{5}\right)^{1.514}(15) \mathrm{a}=\frac{1.6}{100} \mathrm{I}+0.5(16)
$$

- ETP $(\mathrm{mm})$ : l'évapotranspiration moyenne du mois $(\mathrm{m}=1$ à 12$)$ en $\mathrm{mm}$,

- $\mathbf{T}$ : températures moyenne mensuel, ${ }^{\circ} \mathrm{C}$

- I : indice thermique mensuel

- $\mathbf{a}$ : fonction complexe de I

- F : facteur de correction dépendant de latitude, du mois et tenant compte de la durée d'insolation. Ses valeurs sont données sous forme tabulaire. 


\section{- Calcul du bilan hydrique}

Dans le cadre de cette étude, la méthode de Thornthwaite et de FAO 56 Penman - Monteih ont été utilisées pour le calcul du bilan hydrique. Le choix de ces deux méthodes est basé sur le fait qu'elles prennent en compte plusieurs paramètres climatiques (pour la méthode de FAO 56 PenmanMonteith), la simplicité de la méthode (Thornthwaite) et les résultats satisfaisants obtenus par beaucoup d'auteurs (Moctar, 2008; Bouteldjaoui et al., 2011; Abdou Babaye, 2012 ;Madioune, 2012; Mjejra 2015).

\section{$\rightarrow$ Principe de la méthode de Thornthwaite}

Le modèle conceptuel de la méthode de Thornthwaite (1965) se base sur les hypothèses ci-après :

$>$ La zone non saturée du sol est considérée comme un stock rechargeable en eau. Cette hypothèse permet de se décharger de la complexité des écoulements en milieux poreux partiellement saturés;

$>$ La capacité maximale de stock en eau de la zone non-saturée est connue. Cette hypothèse peut se justifier par le fait que le sol ne peut pas contenir plus d'eau que celle équivalent à sa saturation;

$>$ Il ne peut y avoir infiltration vers la nappe ou ruissellement que lorsque cette capacité maximale du stock en eau est satisfaite.

A partir de ces hypothèses, l'estimation de l'évapotranspiration réelle et de ruissellement sont faites tout en tenant compte des principes suivants (Réméniéras, 1986; Abdou Babaye, 2012; Madioune, 2012; Kouame et al., 2014) :

- Lorsque la valeur de l'évapotranspiration potentielle (ETP) est inférieure à celles des précipitations, l'ETR ou l'évapotranspiration réelle correspond donc à l'ETP $: \mathrm{ETP} \leq \mathrm{P} \rightarrow \mathrm{ETR}=\mathrm{ETP}$. L'excédent des précipitations va humidifier la zone non saturée du sol jusqu'à atteindre sa capacité maximale connue (stock en eau). Le surplus contribuera à alimenter l'infiltration et le ruissellement. Le stock en eau du sol, appelé aussi réserve utile 'RU', correspond à la réserve en eau du sol utilisable par les plantes (Trouchon et Morlon., 1999). Sa valeur est estimée à l'aide de la réserve facilement utilisable (RFU) qui est une réserve conceptuelle car n'ayant pas de réalité physique (Drouart et Vouillamoz, 1999), même si l'on peut l'assimiler à la quantité d'eau stockée dans les premiers mètres du sol (Salvayre, 1990 et Marsily, 1994 in Dakoure, 2003).

- Par contre, lorsque la valeur de l'évapotranspiration potentielle (ETP) excède celles de précipitations, la pluie efficace sera nulle (ruissellement et infiltration). L'ETR correspond dans ce cas à la somme des précipitations du mois et tout ou partie du stock en eau du 
sol. En fonction de la valeur de ce stock en eau du sol, deux situations peuvent se présenter :

- Si le stock en eau du sol du mois précédent est largement suffisant pour compléter le déficit des précipitations, alors, ETR est égale à l'ETP $(\mathrm{ETR}=\mathrm{ETP})$;

- Au contraire, si le stock en eau du sol ne pas significatif, donc impossible de combler le déficit des précipitations, pour ce cas, l'ETR correspond aux précipitations du mois plus le stock en eau du sol.

Le choix de la valeur de la réserve utile ou stock en eau du sol constitue la chose la plus difficile dans l'application de la méthode de Thornthwaite. En effet, celle-ci dépend à priori de la nature, de la lithologie et de l'épaisseur de la couche superficielle. Elle dépende aussi du climat, de la profondeur du niveau piézométrique et est surtout influencée par le type de couverture végétale. Plusieurs études ont été réalisées dans le but de déterminer la valeur de cette réserve utile. Selon Réméniéras (1986), cette valeur varie de 10 à 20 $\mathrm{mm}$ par $30 \mathrm{~cm}$ de sol en terrain sablonneux et plus de $100 \mathrm{~mm}$ par $30 \mathrm{~cm}$ d'épaisseur de sol en terrain limoneux ou argileux. Elle peut atteindre jusqu'à $300 \mathrm{~mm}$ dans les zones arides (Marsily, 1994).

Dans le but de déterminer la réserve utile, un autre modèle a été proposé par Thornthwaite selon une loi exponentielle négative de la forme (Kouame et al., 2014) :

$$
R U_{t}=R U_{0 .} e^{-\alpha \sum D P}(18)
$$

Avec :

$\mathbf{R U}_{\mathbf{t}}$ : état de réserve à l'instant $\mathrm{t}$;

$\mathbf{R U}_{\mathbf{0}}$ : réserve utile du sol atteinte à la fin de la saison humide.

DP (déficit pluviométrique) = ETP-P

$\boldsymbol{\alpha}:$ constante dépendant de RU. Ses valeurs sont tabulées.

$\mathrm{Au}$ Niger, l'institut de radio-isotope (IRI) de l'université Abdou Moumouni de Niamey montre que la valeur de la réserve utile varie de $60 \mathrm{~mm}$ à $110 \mathrm{~mm}$ (Ousmane, 1988). De ce fait, compte tenu de la nature sableuse du sol, de la faible profondeur des nappes, des résultats d'occupation de sols nous avons considéré dans cette étude, les valeurs variant de 50 à $100 \mathrm{~mm}$, dans le but de déterminer une valeur plus proche de la réalité.

\section{- Estimation de la "pluie efficace"}

Dans le bassin versant de la Korama, il n'existe qu'une seule station de jaugeage sur son ensemble, ce qui rend difficile l'estimation réaliste de ruissellement. Les données de jaugeages disponibles couvrent la période de 1967 à 1981 et de 1994 à 2016. Il a été effectué au pas de temps journalier. La 
partie du bassin versant qui draine l'eau à l'exutoire est caractérisée par une superficie de $750 \mathrm{~km}^{2}$.

Compte tenu des caractéristiques de ce bassin, des données des mesures de jaugeage, du manque des mesures hydrologiques spatialement distribuées, de la couverture végétale obtenue par la carte d'occupation de sol, des formations géologiques de recouvrements (sables argileux, sables latéritiques), de la topographie du terrain, de la nature des sols, le coefficient de ruissellement a été estimé à $0.5 \%$. Néanmoins, cette valeur peut être sous ou surestimer dans certaines parties du bassin. Notons par ailleurs que, l'intensité et la durée de précipitation sont aussi de très important facteurs qu'il ne faut pas négliger dans la détermination de ruissellement. En effet, selon Gallaire (1995 in Abdou Babaye, 2012), au cours des pluies de forte intensité, les Koris se remplissent rapidement et débordent les berges de leurs lits mineurs et parfois même les lits majeurs, pour provoquer des inondations parfois violentes. Alors que, les pluies de faibles intensités sur une longue durée, favorisent l'infiltration au dépend de ruissellement.

\section{Résultats}

\section{III.1. Variabilité des paramètres climatiques}

\section{III.1.1. Variation des moyennes mensuelle de la pluviométrie}

Les moyennes mensuelles des précipitations de la période de 1961 à 2016 (fig. 3) montrent une importante fluctuation au cours de la saison pluvieuse. Par ailleurs, $90 \%$ des précipitations annuelles tombent de Juin à Septembre. Néanmoins, les mois d'Août et de Juillet sont les plus pluvieux avec respectivement $39,18 \%$ et $29,76 \%$ des précipitations moyennes annuelles. En dehors des mois allant de juin à septembre, on enregistre de faible pluie en Mai et en d'Octobre.

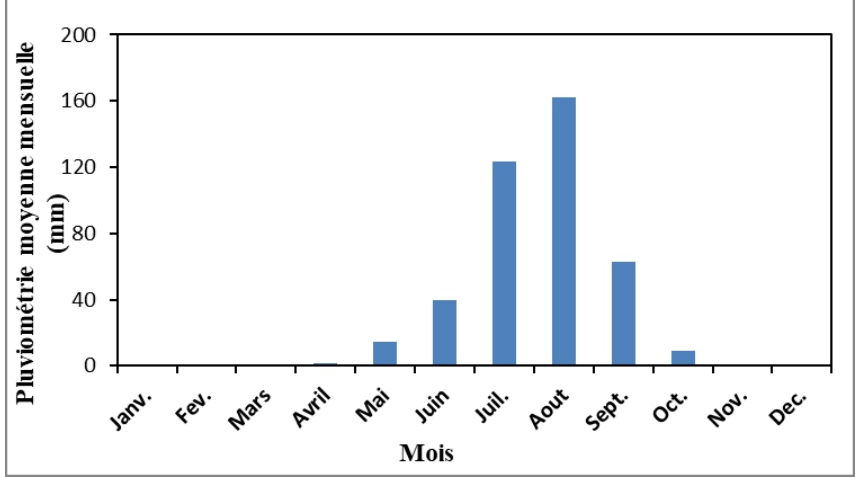

Figure 3: Variation mensuelle des précipitations de 1961 à 2013. 


\section{III.1.2. Variation des moyennes interannuelle de la pluviométrie}

La variabilité pluviométrique étudiée à partir de l'indice de Nicholson et du Filtre passe-bas de Hanning pour la période allant de 1921 à 2016 (fig. 4), a permis de distinguer les trois phases climatiques suivantes :

- une première phase humide s'étalant sur la période de 1921 à 1964, caractérisée par une moyenne pluviométrique interannuelle de $533 \mathrm{~mm}$ avec un écart-type de $110 \mathrm{~mm}$ (tableau 1). Cette moyenne est supérieure à la moyenne interannuelle de la série qui est $464 \mathrm{~mm}$. Au cours de cette phase, l'année 1946 a été excédentaire avec une hauteur moyenne annuelle de $800 \mathrm{~mm}$ et peut être considérée comme exceptionnelle dans toute la série pluviométrique de la période de 1921 à 2016;

- une seconde phase de sécheresse allant 1965 à 1997, caractérisée par des années déficitaires avec une pluviométrie moyenne estimée à 378 $\mathrm{mm}$ et un écart-type de $110 \mathrm{~mm}$ (fig. 4). Pendant cette phase, la moyenne pluviométrique interannuelle est déficitaire de $86 \mathrm{~mm}$ par rapport au module pluviométrique de 1921 à 2016. Par ailleurs, au cours de cette phase, l'année 1983 a été la plus déficitaire de toute la série, avec une hauteur moyenne annuelle de $204 \mathrm{~mm}$ (tableau 1).

- une troisième phase couvrant la période de 1998 à 2016, qui semble être une année normale, avec une moyenne pluviométrique interannuelle de $450 \mathrm{~mm}$ qui est très proche du module pluviométrique de la série (tableau 1).

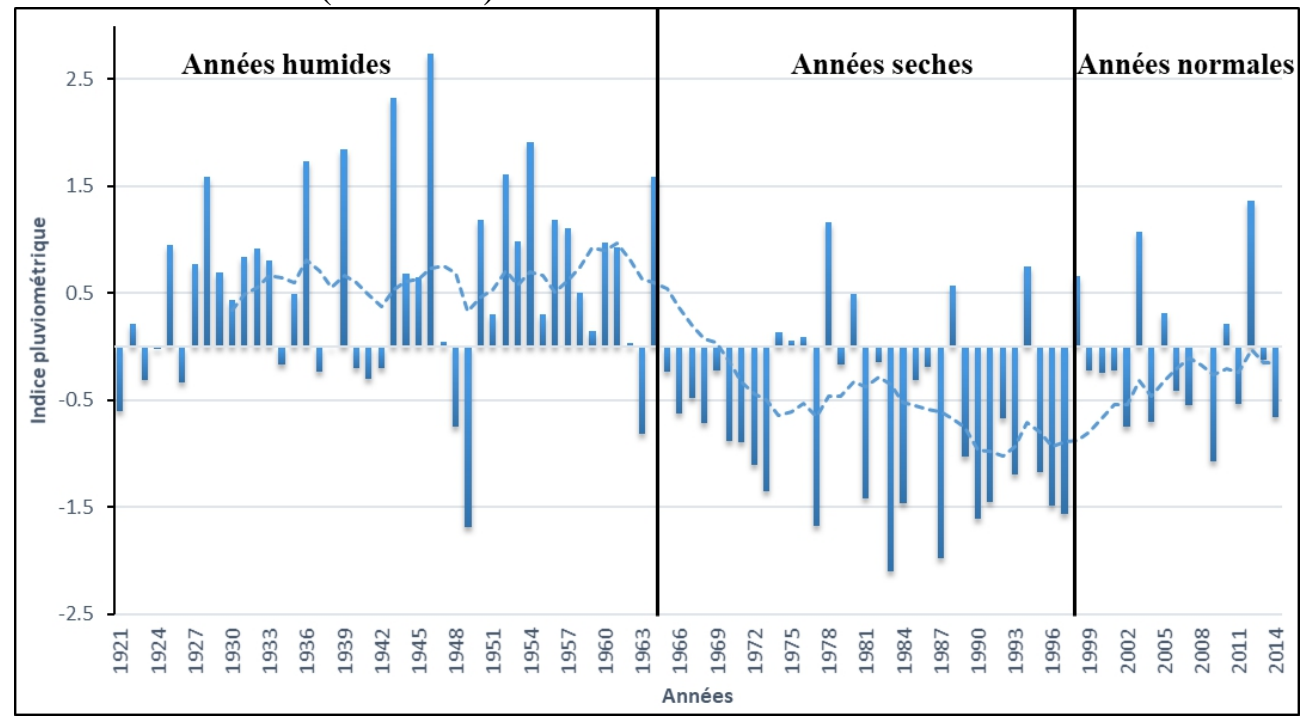

Figure 4: Moyenne mobile sur cinq ans et Indice pluviométrique annuel à la station de Zinder aéroport. 
Tableau I: Caractéristiques des périodes humide, normale et sèche

\begin{tabular}{|l|l|l|l|l|}
\hline Périodes & Moy (mm) & Maxi (mm) & Mini (mm) & Ecart-type (mm) \\
\hline $\begin{array}{l}\text { Humides } \\
(1921-1964)\end{array}$ & 533 & 800 & 256 & 110 \\
\hline $\begin{array}{l}\text { Sèches } \\
(1965-1997)\end{array}$ & 378 & 607 & 204 & 101 \\
\hline $\begin{array}{l}\text { Normales } \\
(1998-2014)\end{array}$ & 450 & 631 & 331 & 81 \\
\hline
\end{tabular}

\section{III.1.3. Détection de ruptures dans la série pluviométrique}

Les résultats de trois tests de ruptures appliquées à la série pluviométrique montrent une seule rupture significative observée en 1965 (fig. 5 et 6 et tableau II). Une seconde rupture, peu significative, a été mise en évidence en 1998 uniquement par le Test de Petitt (fig. 5). Cependant, l'absence des ruptures dans une série pluviométrique n'exclut pas l'absence des variations temporelles des précipitations. Néanmoins celles-ci sont peu significatives (Kanahon et al., 2009; Abdou Babaye, 2012; Bodian, (2014 in Fita et al., 2015).

Les déficits pluviométriques calculés pour les différentes ruptures (tableau III) est de $25 \%$ pour la rupture significative de 1965. Par contre, pour la rupture peu significative, la valeur obtenue correspond à une hausse de $18 \%$ à partir de 1998. Traduisant un retour à des conditions normales des précipitations vers la fin de la décennie 1990. Cependant, ces précipitations annuelles restent inférieures à celles d'avant 1965. Ces observations sont conformes à celles faites par plusieurs auteurs en Afrique de l'Ouest : Sarr et al., 2013 (Sénégal), Lodoun et al., 2013 (Burkina Faso), Ozer et al., 2009 (Niger, dans le bassin du fleuve Niger), Ozer et al., 2014 (Mauritanie).

Tableau II: Caractéristiques des différents tests de rupture

\begin{tabular}{|c|c|c|c|c|c|c|}
\hline Test & Périodes & Rupture & observations & $\begin{array}{c}\text { Moyenne } \\
\text { avant rupture }\end{array}$ & $\begin{array}{c}\text { Moyenne } \\
\text { après rupture }\end{array}$ & C.V \% \\
\hline & $1921-2014$ & 1965 & Significatif & 533 & 402 & 25 \\
\cline { 2 - 7 } Petitt & $1966-2008$ & 1998 & $\begin{array}{c}\text { Peu } \\
\text { Significatif }\end{array}$ & 376 & 442 & 18 \\
\hline Lee & $1921-2014$ & 1965 & Significatif & 533 & 402 & 25 \\
\hline Hubert & $1921-2014$ & 1965 & Significatif & 533 & 402 & 25 \\
\hline
\end{tabular}




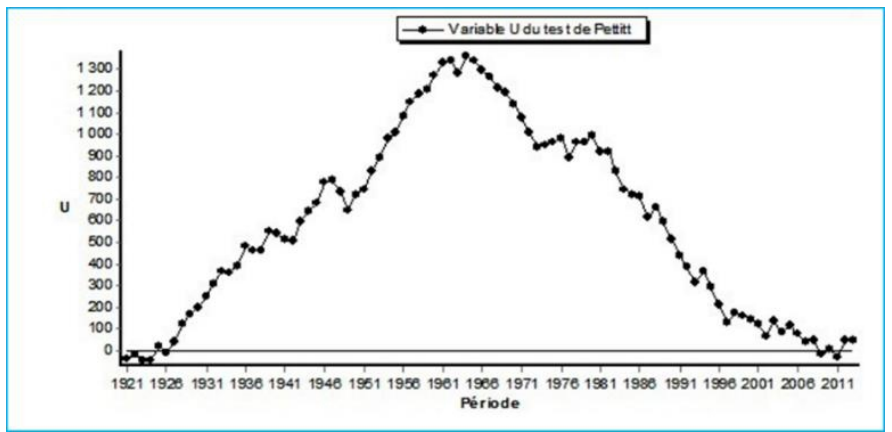

Figure 5: Test de Petitt appliquée à la série pluviométrique (1921 à 2013) de la station de Zinder aéroport.

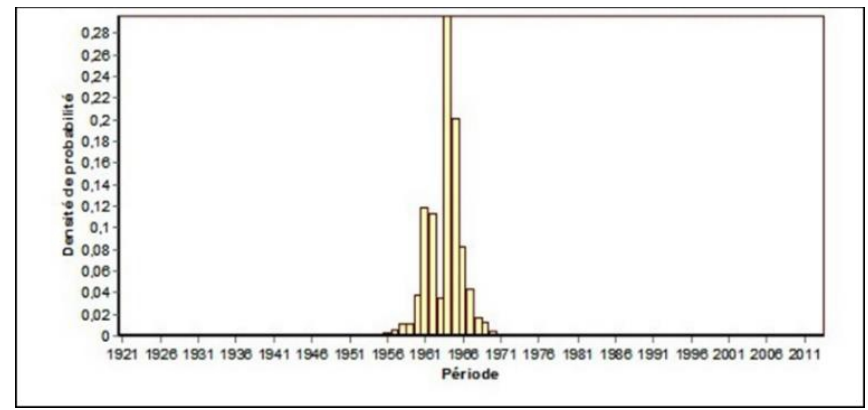

Figure 6: Test de Lee appliquée à la série pluviométrique de la station de Zinder aéroport

Tableau 3: Test de rupture appliquée à la série pluviométrique de la station de Zinder aéroport.

Niveau de signification du test de Scheffé : 1\%

\begin{tabular}{|l|l|l|l|}
\hline Début & Fin & Moyenne $(\mathrm{mm})$ & Ecart-type $(\mathrm{mm})$ \\
\hline 1921 & 1964 & 533 & 110 \\
\hline 1965 & 2014 & 402 & 100 \\
\hline
\end{tabular}

\section{III.1.4. Evolution interannuelle des températures}

La température varie très largement de $22,66^{\circ} \mathrm{C}$ à $33,79^{\circ} \mathrm{C}$. Elle est relativement forte pendant les mois d'Avril et Mai, en cours de saison sèche et en Octobre à la fin de la saison pluvieuse. Cette température baisse de Novembre en février, saison froide et en Juillet-Aout, saison pluvieuse. La température est indicatrice du pouvoir évaporant de l'air et par conséquent un facteur déterminant dans l'évaluation du bilan hydrique (Melloul et al., 2009). On observe une légère augmentation des températures (maximum, minimum et moyenne) au cours de la période de 1961 à 2013 (fig. 7). 


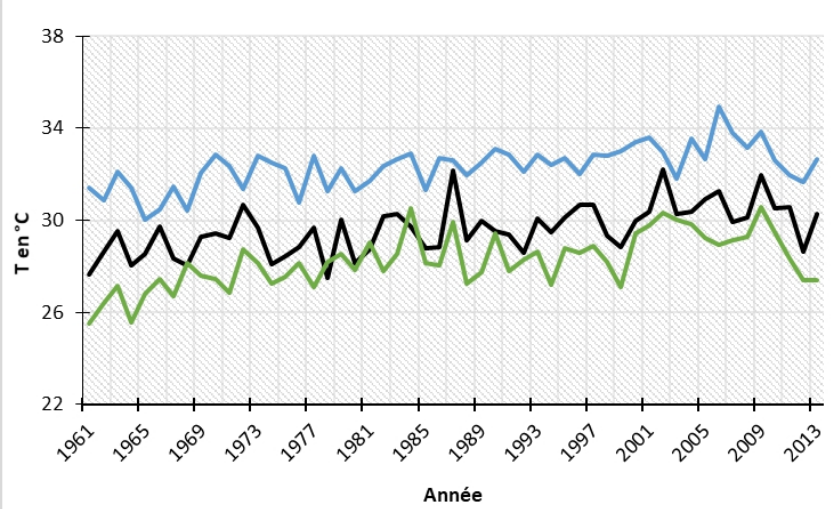

Figure 7: Evolution des températures maximales (en bleu), moyennes (en noir) et minimales (en vert) (1961-2013)

\section{III.2. Evaluation de la pluie efficace avec l'ETP de Thornthwaite}

Le bilan hydrologique calculé par la méthode de Thornthwaite, au pas de temps mensuel, a permis de déterminer les valeurs de la pluie efficace sur la période de 1961 à 2013 :

\section{$\rightarrow$ Pour la phase humide (1961-1964),}

La région a reçu une moyenne interannuelle des précipitations des 518 $\mathrm{mm}$ et une ETP moyenne interannuelle de $1134 \mathrm{~mm}$. La valeur de l'évapotranspiration réelle (ETR) obtenue est de $359 \mathrm{~mm}$, représentant $70 \%$ de la lame d'eau précipitée. Pour une RFU (réserve facilement utilisable) égale à $50 \mathrm{~mm}$, la valeur de la pluie efficace correspond à $158 \mathrm{~mm}$. Par ailleurs, en considérant, le coefficient de ruissellement de 1,55\% des précipitations annuelles, la valeur de ruissellement annuelle est de $8 \mathrm{~mm}$ et la lame d'eau infiltrée correspondra à $150 \mathrm{~mm}$, soit $23 \%$ de pluie tombée (tableau IV). Ainsi, l'équation du bilan hydrologique est de :

$518 \mathrm{~mm}(\mathbf{P})=359 \mathrm{~mm}(\mathbf{E T R})+158 \mathrm{~mm}(\mathbf{I}+\mathbf{R})$ ou

$518 \mathrm{~mm}(\mathbf{P})=359 \mathrm{~mm}(\mathbf{E T R})+150 \mathrm{~mm}(\mathbf{I})+8 \mathrm{~mm}(\mathbf{R})(9)$

Tableau IV: Résultats du bilan hydrologique pour la période humide (1961-1964)

\begin{tabular}{|c|c|c|c|c|c|c|}
\hline \multirow[b]{2}{*}{ MOIS } & \multirow{2}{*}{$\begin{array}{l}\text { Pluie } \\
(\mathbf{m m})\end{array}$} & \multirow{2}{*}{$\begin{array}{l}\text { ETP } \\
(\mathbf{m m})\end{array}$} & \multirow{2}{*}{$\begin{array}{l}\text { ETR } \\
(\mathbf{m m})\end{array}$} & \multicolumn{3}{|c|}{ Valeur de la pluie efficace pour } \\
\hline & & & & RU $=50 \mathrm{~mm}$ & $\mathrm{RU}=75 \mathrm{~mm}$ & RU $=100 \mathrm{~mm}$ \\
\hline $\begin{array}{l}\text { Moyennes } \\
\text { annuelles }\end{array}$ & 518 & 1134 & 360 & 158 & 136 & 120 \\
\hline & & \multicolumn{2}{|c|}{$\begin{array}{l}\text { Infiltration } \\
(\mathbf{m m})\end{array}$} & 150 & 128 & 112 \\
\hline
\end{tabular}

\section{$\rightarrow$ Pour la période sèche (1965-1997)}

La moyenne pluviométrique interannuelle est de $338 \mathrm{~mm}$ et l'ETP moyenne interannuelle représente $1121 \mathrm{~mm}$ (tableau 5). La valeur de l'ETR calculée pour cette période est de $326 \mathrm{~mm}$, soit une pluie efficace de $57 \mathrm{~mm}$ se répartissant entre le ruissellement et l'infiltration. Cependant en soustrayant 
la valeur du ruissellement, l'infiltration, qui correspondrait à la part de la recharge, est de $51 \mathrm{~mm}$, soit $15 \%$ de la hauteur moyenne des précipitations tombées dans le bassin. Ainsi, l'équation du bilan pour cette période est de : $383 \mathrm{~mm}(\mathrm{P})=325 \mathrm{~mm}(\mathrm{ETR})+57 \mathrm{~mm}(\mathrm{I}+\mathrm{R})$ ou (équation $\left.\mathrm{n}^{\circ} 10\right)$ $383 \mathrm{~mm}(\mathrm{P})=325 \mathrm{~mm}(\mathrm{ETR})+6 \mathrm{~mm}(\mathrm{R})+51 \mathrm{~mm}(\mathrm{I})$

Tableau 5: Résultats du bilan hydrologique pour la période sèche (1965-1997)

\begin{tabular}{|c|c|c|c|c|c|c|}
\hline \multirow[b]{2}{*}{ Mois } & \multirow{2}{*}{$\begin{array}{l}\text { ETP } \\
(\mathbf{m m})\end{array}$} & \multirow{2}{*}{$\begin{array}{l}\text { PLUIE } \\
(\mathbf{m m})\end{array}$} & \multirow{2}{*}{$\begin{array}{l}\text { ETR } \\
(\mathbf{m m})\end{array}$} & \multicolumn{3}{|c|}{ Valeur de la pluie utile pour } \\
\hline & & & & RU $=50 \mathrm{~mm}$ & $R U=75 \mathrm{~mm}$ & RU $=100 \mathrm{~mm}$ \\
\hline $\begin{array}{l}\text { Moyenne } \\
\text { annuelle }\end{array}$ & 1121 & 382 & 325 & 57 & 49 & 36 \\
\hline & \multicolumn{2}{|c|}{ Infiltration (mm) } & & 51 & 43 & 30 \\
\hline
\end{tabular}

\section{$\rightarrow$ Pour la période normale (1998-2013)}

Le bilan hydrologique met en évidence, le retour de la pluviométrie (tableau 6). Celui-ci a engendré une augmentation de la réserve utile qui atteint $98 \mathrm{~mm}$ soit environ $22 \%$ du cumul pluviométrique annuelle. Cette réserve utile représente $7 \mathrm{~mm}$ de la quantité d'eau ruisselée et $91 \mathrm{~mm}$ de la lame d'eau infiltrée soit environ $20 \%$ de la lame d'eau précipitée. L’équation du bilan pour la période normale s'établit :

$450 \mathrm{~mm}(\mathrm{P})=352 \mathrm{~mm}(\mathrm{ETR})+98 \mathrm{~mm}(\mathrm{I}+\mathrm{R}) \mathrm{Ou} \quad$ (équation $\left.\mathrm{n}^{\circ} 11\right)$

$450 \mathrm{~mm}(\mathrm{P})=352 \mathrm{~mm}(\mathrm{ETR})+7 \mathrm{~mm}(\mathrm{R})+91 \mathrm{~mm}(\mathrm{I})$

Tableau 6: Résultats du bilan hydrologique pour la période 1998-2013

\begin{tabular}{|c|l|l|l|l|l|l|}
\hline & Pluie & & ETR & \multicolumn{3}{|c|}{ Valeurs de la pluie utile pour : } \\
\cline { 5 - 7 } MOIS & $(\mathbf{m m})$ & ETP $(\mathbf{m m})$ & $(\mathbf{m m})$ & RU=50 $\mathbf{~ m m}$ & RU=75 $\mathbf{~ m m}$ & RU=100mm \\
\hline $\begin{array}{l}\text { Moyennes } \\
\text { annuelles }\end{array}$ & 450 & 1145 & 352 & 98 & 78 & 54 \\
\hline & & & 91 & 71 & 47 \\
\hline
\end{tabular}

Ces résultats montrent les variabilités mensuelles et interannuelles de la recharge en fonction des hauteurs des précipitations. Par ailleurs, les hauteurs des précipitations mensuelles et/ou les valeurs mensuelles de l'ETP de Thornthwaite conditionnent considérablement les valeurs de l'ETR et de la recharge. Néanmoins, on peut relever les faits suivants (tableaux $4 ; 5 ; 6 ; 7$ et fig. 8) :

$\checkmark$ Certaines années à pluviométrie annuelle importante montrent des valeurs faibles ou nulles de la recharge. C'est le cas de 2005, caractérisée par une pluviométrie annuelle de $550 \mathrm{~mm}$ dont la valeur de la recharge est quasi nulle. Par contre, la hauteur de la recharge a été estimée à $17,1 \mathrm{~mm}$ pour une hauteur pluviométrique annuelle de $435 \mathrm{~mm}$ en 2001.

Ces résultats montrent qu'il est nécessaire d'approfondir la connaissance sur l'influence d'autres facteurs susceptibles d'intervenir dans la recharge.

A partir de certaines hauteurs de précipitations (fig. 8) qui semble se situer en dessous de $350 \mathrm{~mm}$, toute recharge directe parait impossible ; 
$\checkmark$ Malgré les faibles valeurs de la RFU traduisant des recharges faibles ou nulles, il a été constaté une remontée très significative du niveau piézométrique. Ces résultats semblent confirmer la recharge indirecte des nappes dans ces zones arides à semi-arides ;

$\checkmark$ Les valeurs de la recharge obtenues au cours de la phase humide ; sèche et normale sont respectivement de $158 \mathrm{~mm}, 57 \mathrm{~mm}$ et $98 \mathrm{~mm}$. Par conséquent, la lame d'eau efficace varie en fonction des hauteurs pluviométriques interannuelles.

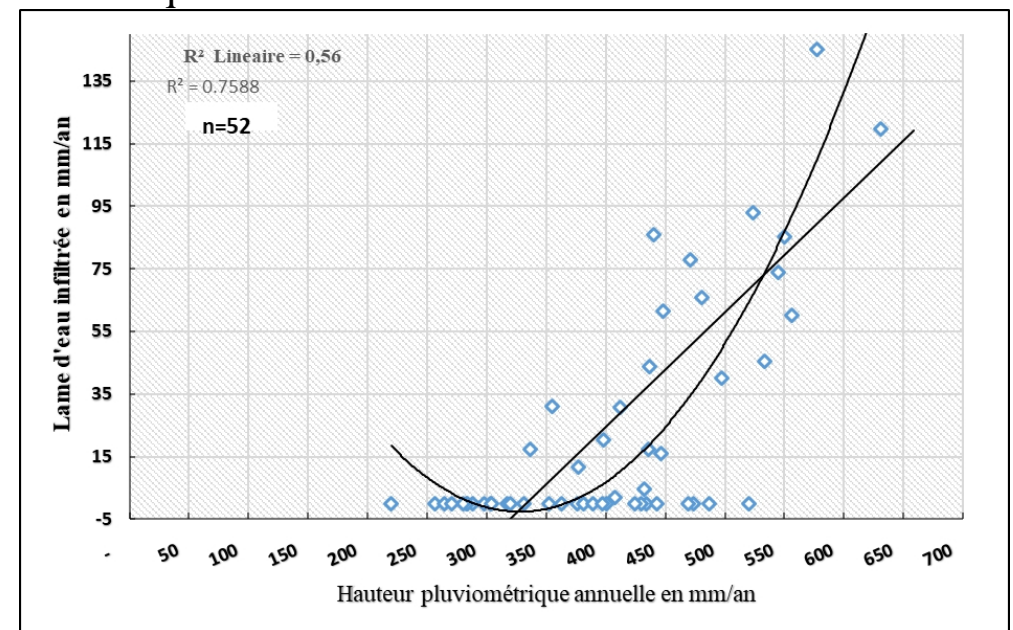

Figure 8: Infiltration moyenne annuelle en fonction des précipitations moyennes annuelles sur la période de 1961-2013.

\section{III.3. Evaluation de la pluie efficace avec l'ETP de PENMAN}

Le calcul du bilan hydrologique avec l'ETP de Penman a concerné la période de 2000 à 2009 (tableau VII). Seule l'année 2003, caractérisée par une pluviométrie annuelle de $550 \mathrm{~mm}$ montre une pluie efficace de $6 \mathrm{~mm}$ pour une valeur de la RFU=50 mm. Ainsi, en considérant, le coefficient de ruissellement de $1,55 \%$ des précipitations, l'infiltration est quasi-nulle. Cependant, l'équation du bilan devient :

$550 \mathrm{~mm}($ Pluie $)=544 \mathrm{~mm}($ ETR $)+6 \mathrm{~mm}(\mathrm{I}+\mathrm{R})$ ou (équation $\left.\mathrm{n}^{\circ} 13\right)$

$550 \mathrm{~mm}($ Pluie $)=544 \mathrm{~mm}($ ETR $)+6 \mathrm{~mm}(\mathrm{R})+0 \mathrm{~mm}(\mathrm{I})$ avec $\mathrm{I} \approx 0$

Ces résultats semblent montrer pour l'équation du bilan établit à partir de l'ETP de Penman, la recharge semble nulle même avec des hauteurs pluviométriques atteignant $550 \mathrm{~mm}$.

Tableau VII: Valeur de la pluie utile sur la période de $2000-2009$.

\begin{tabular}{|l|l|l|l|l|l|l|}
\hline & Pluviométrie & $\begin{array}{l}\text { ETP } \\
\text { Année }\end{array}$ & $(\mathbf{m m})$ & RU=50mm & RU=75mm & RU=100mm \\
\cline { 4 - 6 } & $(\mathbf{m m})$ & \multicolumn{2}{|c|}{ Valeurs de la pluie utile (mm) } \\
\hline 2000 & 432,7 & 2533,8 & 0 & 0 & 0 \\
\hline 2001 & 435,4 & 2117,9 & 0 & 0 & 0 \\
\hline 2002 & 388,9 & 2169,1 & 0 & 0 & 0 \\
\hline 2003 & 549,7 & 2154,0 & 6,2 & 0 & 0 \\
\hline
\end{tabular}




\begin{tabular}{|l|l|l|l|l|l|}
\hline 2004 & 376,8 & 2621,2 & 0 & 0 & 0 \\
\hline 2005 & 520,2 & 2609,0 & 0 & 0 & 0 \\
\hline 2006 & 411,9 & 2642,0 & 0 & 0 & 0 \\
\hline 2007 & 397,7 & 2623,5 & 0 & 0 & 0 \\
\hline 2008 & 408,0 & 2500,9 & 0 & 0 & 0 \\
\hline 2009 & 330,9 & 2585,1 & 0 & 0 & 0 \\
\hline
\end{tabular}

\section{III.4. Comparaison des résultats de la méthode de Thornthwaite et celle de Penman}

La comparaison des résultats de la méthode de Thornthwaite et celle de Penman concerne l'année 2003. Où il existe de valeurs de pluie efficace supérieure à zéro pour ces deux méthodes. Ainsi, les valeurs de la pluie efficaces obtenues par la méthode de Thornthwaite sont plus importantes que celles obtenus par la méthode de Penman (tableau 8). Cette différence s'explique par le fait que la valeur de l'ETP de Penman est deux (02) fois plus élevée que celle de Thornthwaite.

Tableau VIII: Comparaisons de résultats obtenus par les méthodes de Thornthwaite et de Penman pour l'année 2003.

\begin{tabular}{|c|c|c|c|c|c|}
\hline & \multirow{2}{*}{$\begin{array}{ll}\begin{array}{l}\text { Pluie } \\
(\mathrm{mm})\end{array} & \text { totale } \\
\end{array}$} & \multirow{2}{*}{$\begin{array}{l}\text { ETP } \\
(\mathbf{m m})\end{array}$} & \multicolumn{3}{|c|}{ Pluie utile en fonction de la valeur de RU } \\
\hline & & & RU $=50 \mathrm{~mm}$ & RU $=75 \mathrm{~mm}$ & RU $=100 \mathrm{~mm}$ \\
\hline Thornthwaite & 550 & 1140 & 62,5 & 31,2 & 6,2 \\
\hline Penman & 550 & 2154 & 178,75 & 153,75 & 128,75 \\
\hline Rapport & $\approx 1,0$ & $\approx 0,5$ & $\approx 2,9$ & $\approx 4,9$ & $\approx 20,8$ \\
\hline
\end{tabular}

\section{Discussion}

\section{IV.1. Analyse De La Variation Climatique}

Les différentes phases climatiques observées montrent que les pays sahéliens ont été bel et bien des théâtres de changement climatique ayant occasionné des conséquences drastiques sur l'environnement biophysique et les populations (fig.4; tableau I). Ce qui a été aussi confirmée par les résultats de tests de ruptures (fig. 5 et 6 ; tableaux II et III). Ces derniers sont conformes à ceux de plusieurs auteurs ayant montré des brusques changements (diminution) des précipitations en Afrique de l'Ouest à la fin de 1960 (Paturel et al., 1996; 1998 ; Ozer et al., 2009). Néanmoins, les auteurs ont observé ces changements à des dates variables. Ainsi, dans les pays riverains du Golfe de Guinée, Paturel et al., (1996) ont montré que les dates des changements observés se situent majoritairement autour de 1970. Par contre, pour quelques stations (Béoumi), ce changement avait eu lieu en 1965. Alors que pour d'autres, il n'est intervenu que vers 1980 (Abidjan). Au Niger, particulièrement 
dans la partie Orientale, Ozer et al., (1999) montrent que la crise climatique a bel et bien débuté à la fin des années 1960 de manière quasi simultanée.

\section{IV.2. Analyse du bilan hydrologique}

$\mathrm{Au}$ vu des résultats sur ces trois périodes, la recharge de la nappe phréatique varie de manière spatiotemporelle et selon les conditions climatiques. Toutefois, les équations de ce bilan permettent de mettre en évidence l'importance de l'infiltration sur le ruissellement dans la zone d'étude. Ce phénomène pourrait être lié au caractère essentiellement sableux de ce bassin, confirmant ainsi, les résultats des études précédentes ayant relevé la perméabilité des formations de recouvrement dudit bassin (Plote, 1961; Biscaldi, 1962; Henri, 1995). En effet, les aquifères de sables récents ont montré des coefficients de transmissivité de $4.10^{-3} \mathrm{~m}^{2} / \mathrm{s}$ à $10^{-2} \mathrm{~m}^{2} / \mathrm{s}$, d'emmagasinement compris entre 2,5 à 11,5\% (Plote, 1962). Par ailleurs, l'analyse granulométrique effectuée sur ces sables de recouvrement de la vallée de Gogo a donné un coefficient de Hazen égal à 3, traduisant ainsi, une excellente perméabilité (Plote, 1961 in Biscaldi, 1962).

\section{Conclusion}

Ces principaux résultats montrent que la région a été un théâtre de changement climatique caractérisé par l'alternance des trois phases: humides, sèches et normales. Cette variabilité climatique a pour conséquence sur les eaux souterraines, l'importante diminution de taux de recharge pendant la période sèche. Néanmoins, au cours de la période normale, suite au retour des précipitations qu'a connu la région, on assiste à un relèvement de taux de recharge. Toutefois, cette augmentation du taux de recharge est trop faible quand on le compare à celle de la période humide et fasse à la demande accrue de besoin en eau pour l'approvisionnement de la population, l'irrigation et l'élevage qui s'effectuent dans la zone.

Par ailleurs, les valeurs de l'infiltration sont toujours supérieures à celles de ruissellement, ceci résulte du caractère essentiellement sableux de ce bassin. Enfin, il ressort que la détermination de l'ETR par la méthode de Thornthwaite a été une approche très efficace. En effet, le résultat obtenu par l'ETP de Thornthwaite pour ce bilan semble plus réaliste que ceux obtenues à partir de l'ETP de Penman.

Compte tenu, des différentes activités et fasse au phénomène de la variabilité climatique, il s'avère nécessaire d'entreprendre une étude sur la piézométrie afin de mieux quantifier la recharge.

Comment les variations des différents paramètres étudiés agissent t-elles sur le niveau de la nappe la plus exploitée de votre zone d'étude.

L'alimentation de cette nappe s'effectue principalement par l'infiltration direct des eaux de précipitations et par drainage de cours d'eau 
de surface. Lorsque les précipitations mensuelles, annuelles ou interannuelles sont importantes, cela traduirait un taux de recharge élevé dans la nappe. La température, l'ETP et l'ETR sont des paramètres climatiques interdépendants. Ils varient dans le même sens. Compte tenu de la nature sableuse du recouvrement, une forte évaporation dans la zone d'étude pourrait traduire directement une baisse de niveau de la nappe et accélérer un assèchement rapide de cours d'eau de surface.

\section{References:}

1. Abdou Babaye Maman Sani (2012) : Evaluation de ressources en eau souterraine dans le bassin de Dargol (Liptako-Niger). Thèse de doctorat de l'Université Abdou Moumouni de Niamey.

2. Assani Ali 1999: Analyse de la variabilité temporelle des précipitations (1916-1996) à Lubumbashi (Congo-Kinshasa) en relation avec certains indicateurs de la circulation atmosphérique (oscillation australe) et océanique (El Niño/La Niña). Sécheresse, 10(4), pp 245-252.

3. E.D Fita., A Ombolo., G.M. Ewodo., S. Chouto., E.A. Bineli., M.J.E. Abate., (2015): Caractérisation de la variabilité spatio-temporelle des précipitations dans la zone soudano-sahélienne du Cameroun au cours des cinq dernières décennies. Afrique Science 11(4), 331 - 348 pp.

4. P. Hubert., P.J. Carbonnel., et A. Chaouche., 1989: Segmentation des séries hydrométéorologiques-Application à des séries de précipitations et de débits de l'Afrique de l'Ouest. Journal of Hydrology, 110, pp 349-367.

5. O.F Kanohin., Saley Maman Bachir., I. Savané., 2009 : Impacts de la variabilité climatique sur les ressources en eau et les activités humaines en zone tropicale humide: Cas de la région de Daoukro en Côte D'ivoire. European Journal of Scientific Research Vol.26 No.2, pp.209-222.

6. Kingumbi., Z. Bergaoui., J. Bourges., P. Hubert., R. Kallel,. (2000) : Etude de l'évolution des séries pluviométriques de la Tunisie Centrale.file:///C|/Apacheroot/htdocs/medweb/WEBdocuments/kingumbi.htm.

7. P. Ozer., C.Y. Hountondji., O.M. Laminou., 2009: Évolution des caractéristiques pluviométriques dans l'est du Niger de 1940 à 2007. Geo-Eco-Trop, 33, pp 11-30.

8. P. Ozer., C.Y Hountondji., J. Gassani., B. Djaby., F. De Longueville., 2014: Évolution récente des extrêmes pluviométriques en Mauritanie (1933-2010). XXVIIe Colloque de l'Association Internationale de Climatologie, Université de Bourgogne, Dijon, France, pp 394-400. 
9. E.J. Paturel., E. Servat., B. Kouamé., F.J. Boyer., H. Lubes., et M.J. Masson., 1995: Procédures d'identification de « ruptures » dans des séries chronologiques -modification du régime pluviométrique en Afrique de l'Ouest non sahélienne. IAHS Publ. n² 238. 1996, pp 99110. Actes de la conférence de Paris, mai 1995.

10. E.J. Paturel., H. Lubes,. E. Servat., O.M. Delattre., 1996: Etude de séries pluviométriques de longue durée en Afrique de l'Ouest et Centrale non sahélienne. XIIème Journées hydrologiques de l'Orstom, Montpellier, 10-11 oct.1996

11. E.J. Paturel., E. Servat., B. Kouamé., H. Lubès., M. Ouedraogo,. Masson J.M., 1997: Climatic variability in humid Africa along the gulf of Guinea. Part II : an integrated regional approach. Journal of Hydrology, 191, pp 16-36.

12. Soro Tanina. D., Kouakou B. D., Kouassi A.E., Soro G., Kouassi M. A., Kouadio E. K., S.M.O. Yéi., N. Soro,. 2006: Hydroclimatologie et dynamique de l'occupation du sol du bassin versant du Haut Bandama à Tortiya (Nord de la Côte d'Ivoire). Vertigo - la revue électronique en sciences de l'environnement, Vol $13 \mathrm{~N}^{\circ} 3$.

13. M. Sophocleous (2004): Groundwater recharge and the water budgets of the Kansas High Plains and related aquifers. Kansas Geological Survey Bulletin 249. 102 p. 